\title{
Peritoneal Endometriosis: Two-Dimensional and Three-Dimensional Evaluation of Typical and Subtle Lesions ${ }^{a}$
}

\author{
JACQUES DONNEZ, MICHELLE NISOLLE, \\ AND FRANÇOISE CASANAS-ROUX \\ Infertility Research Unit \\ Department of Gynecology \\ Catholic University of Louvain \\ Cliniques Universitaires St. Luc \\ Avenue Hippocrate 10 \\ B-1200 Brussels, Belgium
}

Endometriosis most commonly affects the pelvic peritoneum close to the ovaries, including the uterosacral ligaments, the ovarian fossa peritoneum, and the peritoneum of the cul-de-sac.

The increased diagnosis of endometriosis at laparoscopy can be explained by the increased experience and ability of the surgeon to detect such subtle lesions. The greatest change has been in the case of "subtle" lesions, which increased from $15 \%$ in 1986 to $65 \%$ in 1988 . The diagnosis of peritoneal endometriosis at the time of laparoscopy is often made by the observation of typically puckered black or bluish lesions. There are, in addition, numerous subtle appearances of peritoneal endometriosis.

These lesions, frequently nonpigmented, were diagnosed as endometriosis following confirmation by biopsy by Jansen and Russell in 1986.

\section{Typical Lesions}

The typical black peritoneal endometriotic lesion results from tissue bleeding and retention of blood pigment producing brown discoloration of tissue.

Puckered black lesions are a combination of glands, stroma and intraluminal debris.

\section{Evolution}

The macroscopic appearance of ectopic endometrium is probably dependent upon the longevity of the process. Viable cells may implant and the initial appearance may be an irregularity or discoloration of the peritoneal surface-the earliest sign being hemosiderin staining of the peritoneal surfaces. Initially, these lesions

\footnotetext{
a Partially supported by grants from the Fonds de la Recherche Scientifique (Belgique) and from Ipsen Biotech (France).
} 
TABLE 1. Different Appearances of Peritoneal Endometriosis

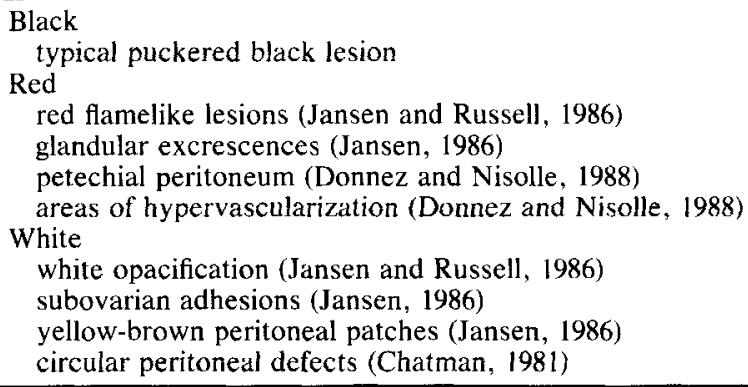

may appear hemorrhagic, but menstrual shedding from a viable endometrial implant initiates an inflammatory reaction which provokes a scarification process which, in turn, encloses the implants. The presence of entrapped menstrual debris is responsible for the typical black or bluish appearance. If the inflammatory process obliterates or devascularizes the endometrial cells, eventually this discoloration disappears. A white plaque of old collagen is all that remains of the ectopic implant. Scarring of the peritoneum around endometrial implants is a typical finding. In addition to encapsulating an isolated implant, the scar may deform the surrounding peritoneum or result in the development of adhesions.

\section{Subtle Appearances}

Sometimes the subtle endometriotic lesions can be the only lesions seen at laparoscopy. The subtle forms are more common and may be more active than the puckered black lesions (TABLE 1).

The nonpigmented endometriotic peritoneal lesions include essentially the following:

\section{Red Lesions}

1) Red flamelike lesions of the peritoneum or red vesicular excrescences more commonly affecting the broad ligament and the uterosacral ligaments. Histologically, red flamelike lesions and vesicular excrescences are due to the presence of active endometriosis surrounded by stroma.

2) Glandular excrescences on the peritoneal surface which in color, translucency and consistency closely resemble the mucosal surface of the endometrium seen at hysteroscopy. Biopsy reveals the presence of numerous endometrial glands.

3) Areas of petechial peritoneum or areas with hypervascularization were diagnosed as endometriosis in our recent study. These lesions resemble the petechial lesions resulting from the manipulation of the peritoneum or from hypervascularization of the peritoneum. They most generally affect the bladder and the broad ligament, and histologically, red blood cells are numerous and endometrial glands are very rare. 


\section{White Lesions}

1) White opacification of the peritoneum which appears as peritoneal scarring or as circumscribed patches, often thickened and sometimes raised. Histologically, white opacified peritoneum is due to the presence of an occasional retroperitoneal glandular structure and scanty stroma surrounded by fibrotic tissue or connective tissue.

2) Subovarian adhesions or adherence between the ovary and the peritoneum of the ovarian fossa, which are distinctive from adhesions characteristic of previous salpingitis or peritonitis. Histologically, connective tissue with sparse endometrial glands was found.

3) Yellow-brown peritoneal patches resembling "café au lait" patches. The histological characteristics are similar to those observed in white opacification, but in the yellow-brown patches the presence of the blood pigment hemosiderin among the stroma cells produces the "café au lait" color.

4) Circular peritoneal defects as described by Chatman. Serial section demonstrates the presence of endometrial glands in more than $50 \%$ of cases. Peritoneal endometriosis can thus be found in the visually normal peritoneum of infertile women with or without associated endometriosis.

\section{Morphometric Study of Vascularization}

Vascularization of endometriotic implants is probably one of the most important factors of growth and invasion of endometrial glands in other tissue. A stereometric analysis was applied in order to study precisely the vascularization in peritoneal endometriotic foci.

We evaluated histologically the vascularization of typical peritoneal endometriosis and its modifications according to the macroscopic appearance of peritoneal endometriosis.

A 2-D image analysis program set on Vidas computer (Kontron Bildanalyse $\mathrm{GmBH}$, Eching, Germany) was completed by the interactive counting of 262,144 points.

All endometriotic lesions $(n=220$ ) were analyzed field by field using the objective $40 \times$ of an Axioskop light microscope (Zeiss, Oberkochen, Germany) and a television camera (Dag-MTI, Michigan City, IN). The histological features were displayed on a television monitor and stored in the memory for processing by the measuring program. The mean of fields analyzed in each case was $13.3 \pm$ 6.7. Histologic structures of interest such as the stroma, the glandular epithelium and lumen, the capillaries and the lymphocytes were drawn moving a cursor. Each different structure was discriminated and grey level images were transferred to binary images. The interactive measurements of the selected parameters (number of structures, area and perimeter of the structures per field) were appended and stored at the end of an existing data base.

Data management and evaluation were checked according to specific search criteria on the Videoplan (Kontron Bildanalyse GmBH, Eching, Germany) and displayed on the television monitor and printed. In all cases, the mitotic index was calculated as previously described (Donnez et al. 1992) by counting mitotic figures (prometaphase, metaphase, anaphase, and telophase) for 2000 epithelial cells per biopsy. This is the only method available for women because administration of colchicine or tritiated thymidine is not ethical. The contingency table method, the $x^{2}$ (chisquare) test, the $t$ test and the median test were used for statistical analysis. 
TABLE 2. Morphometric Study of Stromal Vascularization

\begin{tabular}{lcccc}
\hline & $\begin{array}{c}\text { Typical Lesions } \\
\text { (Black) } \\
\text { Group Ia } \\
\mathrm{n}=135\end{array}$ & $\begin{array}{c}\text { Red Lesions } \\
\text { Group Ib } \\
\mathrm{n}=35\end{array}$ & $\begin{array}{c}\text { Ghite Lesions } \\
\mathrm{n}=50\end{array}$ & $\begin{array}{c}\text { Treated Typical } \\
\text { Lesions } \\
\text { Group Id } \\
\mathrm{n}=45\end{array}$ \\
\hline $\begin{array}{l}\text { Number of capillaries } / \mathrm{mm}^{2} \\
\text { stroma }\end{array}$ & 243 & 147 & 206 & 225 \\
$\begin{array}{l}\text { Capillary mean } \\
\text { surface }\left(\mu \mathrm{m}^{2}\right)\end{array}$ & $118 \pm 84$ & $234 \pm 192^{*}$ & $78 \pm 43^{* *}$ & $71 \pm 40^{* *}$ \\
$\begin{array}{l}\text { Capillaries } / \text { stroma } \\
\text { relative surface }(\%)\end{array}$ & 2.4 & $3.2^{*}$ & $1.5^{* *}$ & $1.4^{* *}$ \\
\hline
\end{tabular}

* Significantly different from groups Ic and Id.

** Significantly different from group la. Modified from Nisolle et al. 1993.

The results concerning the capillaries are shown in TABLE 2. The number of capillaries per $\mathrm{mm}^{2}$ of stroma, their mean surface area and the surface area ratio (capillaries/stroma) were calculated. The mitotic index was calculated in glandular epithelium and its value was $0.1 \%$ and $0.61 \%$ respectively in group Ia and group Ib. In group Ic (white lesions), no mitosis was observed. The vascularization of typical peritoneal endometriosis was evaluated in 45 patients after GnRHagonist therapy.

Our study demonstrated significant differences between the typical (black or bluish) lesion and the "subtle" lesion. Subtle lesions were classified as red lesions (vesicular, red flamelike and glandular excrescences) and as white lesions (white opacification, yellow-brown patches, circular peritoneal defects). When compared to typical lesion data, the vascularization was found to be significantly higher in red lesions and significantly lower in white lesions. This change was due to an increase (red) or a decrease (white) in the volume occupied by the vessels as proved by both the mean capillary surface area and the ratio of capillaries/stroma surface area. This change is more evident in the group of red lesions in which the number of capillaries $/ \mathrm{mm}^{2}$ was significantly lower than in the other subgroups.

Thus, in the red lesions, the increased level of vascularization is due to a greater number of larger vessels than in the other groups. In white lesions, there was a great number of smaller vessels although the number of capillaries was higher than in red lesions.

Moreover, the mitotic index was also significantly different in the three groups. Mitotic processes permit the maintenance and the growth of peritoneal endometriosis. The absence of mitosis in white lesions proves their low "activity." According to our data, we suggest that there are probably different types of peritoneal endometriotic lesions, in different stages of development. Red flamelike lesions and glandular excrescences are probably the first stage of early implantation of endometrial glands and stroma.

The growth and aggressiveness of endometrial glands in the stroma has recently been demonstrated by a three-dimensional evaluation (Ito et al. 1990). Indeed, in this group, a higher incidence of glands with ramifications was observed when compared to typical and white lesions. The significantly higher stromal vascularization and epithelial mitotic index could be responsible for the invasion of glands and stroma into the ectopic sites.

Our results demonstrated that there was a significant decrease in the vascularization of the endometriotic foci after GnRH agonist therapy. This change was 
not due to a reduction in the number of capillaries in the lesion but to a decrease in the area of the vessels. Indeed, in the treated patients (group Id), a predominance of smaller vessels was observed when compared with the untreated patients (group I). This vascularization decrease, observed histologically, was in accordance with the observations made by laparoscopy after hormonal therapy. Vascular effects of the GnRH agonist have also been demonstrated by Doppler on the uterine arteries (Matta et al., 1988). The hypooestradiolemy induced by GnRH-a could also have an effect on the vascular compartment of the endometriotic stroma.

The reduction in the vascularization after hormonal therapy could account for the decrease in the inflammatory reaction observed around the endometriotic foci.

In conclusion, the evaluation of the stromal vascularization permitted the differentiation and classification of the different appearances of peritoneal endometriosis according to their vascularization level. Our study proves that the "activity" of peritoneal endometriosis is related to the vascularity.

\section{Three-Dimensional (3-D) Architecture of Endometriosis}

In order to elucidate further biological characteristics of peritoneal endometriotic lesions, for example, how they stereologically develop in vivo and how glandular epithelium and stroma are related to the surrounding tissue, a recently advanced stereographic computer-technology was applied for the investigation of threedimensional (3-D) architectures of peritoneal endometriosis.

\section{METHODS}

All biopsy specimens were fixed in formaldehyde and embedded in paraffin. Six micrometer serial sections were stained with Gomori's trichrome and examined on a blind basis, with a Leitz Orthoplan microscope (Leitz, Wetzlar, Germany).

The histological features of the sections were displayed using an Axioskop microscope (Zeiss, Oberkochen, Germany) through a CCD 72 E camera (DageMTI, Michigan City, IN) on a monitor on which two-dimensional (2-D) figures drawn with a digitizer were superimposed using a computer (Vidas, Kontron Bildanalyse $\mathrm{GmBH}$, Eching, Germany). Computer-assisted reconstruction of three-dimensional models was developed with two main aims in mind: (1) to generate a complete multicolored model of a complex structure which can be rotated and viewed from any angle or orientation; (2) to calculate the volumes and surfaces within the 3-D model automatically. The major features of the program include (1) input of serial section data by manual tracing, or automatic contour finding; (2) alignment of sections; (3) editing and reassignment of contours of individual sections; (4) storing contour data in a file; (5) selecting a range of sections and/or a range of elements to be used for reconstruction; (6) reconstructing in a wire frame and/or a solid modelling mode by using parallel projection; (7) rotating reconstruction in the $\mathrm{x}, \mathrm{y}$ and $\mathrm{z}$ plane at variable magnification; (8) viewing inside a model by cutting away part of the reconstruction using an "electronic knife;" (9) calculating surfaces and volumes; (10) plotting reconstruction on a matrix or laser print.

With this program, outlines of glandular structure and endometrial stroma in the serial histological sections were traced by the digitizer, sections were aligned and contour data stored in a file: once all the serial outlines had been digitized 
and stored, reconstructed (3-D) image models of these in vivo structures were displayed on the TV monitor. The 3-D reconstruction could generate a complete multicolored model of the complex structure which could be rotated and viewed from any angle or orientation. Volumes of the reconstructed glandular and stromal structures were obtained by calculation function in the same program. Lumen volume which is the volume attributed to the lumen was also calculated. Ratios of lumen volume/epithelial volume/stromal volume were determined for each specimen. The $\chi^{2}$ test was used for statistical analysis.

\section{RESULTS}

In 42 women who were undergoing laparoscopy for infertility, peritoneal biopsies of 3 to $5 \mathrm{~mm}$ in size were taken from areas of the pelvic peritoneum bearing foci of endometriosis, with a biopsy punch forceps (26-175 DH, Storz, Tuttlingen, Germany). Biopsy was taken from the typical (puckered black) endometriotic implants in all cases.

Group 1 consisted of 26 women with peritoneal endometriosis who had not previously received any hormonal therapy. All of them underwent laparoscopy during the early luteal phase.

Group II consisted of 17 women who had received a gonadotrophin-releasing hormone agonist (GnRH-a) therapy (Zoladex, ICI, Cambridge, UK) for 12 weeks before biopsy. After a well-known initial stimulation of estradiol (E2) secretion, GnRH-a administration resulted in a postmenopausal E2 secretion range (15 \pm 6 $\mathrm{pg} / \mathrm{ml}$ ).

Histologically, all the biopsy specimens showed typical epithelium and stroma of the endometrial type. The reconstructed 3-D image models of the structures in the peritoneal endometriotic lesions were displayed and a pink color was applied for the stroma, a green color for the epithelium and blue color for the lumen.

The 3-D image models were usually indicated as a solid structure; however, models could be displayed as a transparent structure, when they were simultaneously shown with their stromal and epithelial structures.

Stereographically, two types could be easily recognized and classified: (1) The first type is composed of cylinder-like glands without ramifications. The lesion shows a regular distribution of the glandular epithelium in the stromal structure which is also regular (FIG. 1). (2) The second type is composed of glands with ramifications (FIG. 2). Luminal structures are interconnected with one another. Epithelial structures appear like fingers and seem to invade the stroma. The distribution of glandular structures in the stroma is not regular. Many glandular structures formed inside luminal structures whose diameter varied from 22 to $185 \mu \mathrm{m}$.

In all groups, the "external" stromal surface is regular. Like normal uterine epithelial structures, the glandular epithelium has a markedly regular luminal surface. In some cases, the lumen is dilated; in other cases, especially when the ramifications are numerous, the lumen is narrow. The incidence of the first type was $44 \%$ in group I and $46 \%$ in group II. The incidence of the second type was $56 \%$ and $54 \%$, respectively. Volumes of epithelial, stromal and luminal structures were separately measured by computer stereometry and the results are shown in TABLE 3. The stroma/lesion ratio was $62.2 \%$ and $51.8 \%$ in group I and II, respectively. Although there was a decrease in stroma/lesion ratio in group II, when compared to group I, the difference was not significant. The epithelium/lesion 


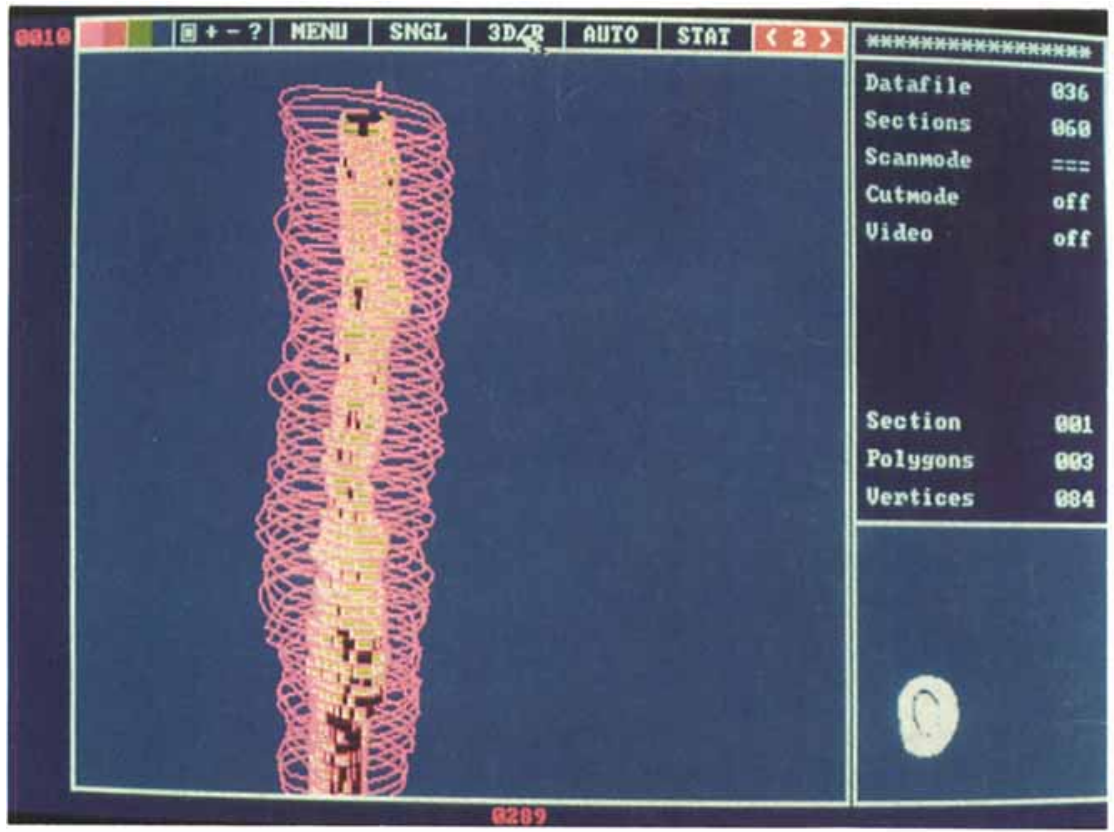

FIGURE 1. Cylinder-like gland. Regular distribution of the glandular epithelium in the stroma.

ratio was $19.9 \%$ and $14.9 \%$, respectively. The lumen/lesion ratio was $13.2 \%$ and $25.2 \%$ in groups I and II, respectively. These values were significantly $(p<0.01)$ different.

\section{COMMENTS}

Recently, computer-stereographic studies of skin tissues have been reported describing the advantages of the computer-generated 3-D models of tissue structures. As far as we know, there has been no publication on the topic of endometriosis using computer-graphic mechanical methods of reconstruction.

When compared with the 3-D models demonstrated in other studies, the present 3-D models seemed to be much better and to show more realistic appearances of structures since the structures of the reconstructed models were colored. Furthermore the transparent display of our 3-D models was excellent for the observation of their inside structures. The present study demonstrates that two different types of endometrial peritoneal lesions can be differentiated: (1) a first type without ramification of the glands; (2) a second type in which glands are ramified and connected. 


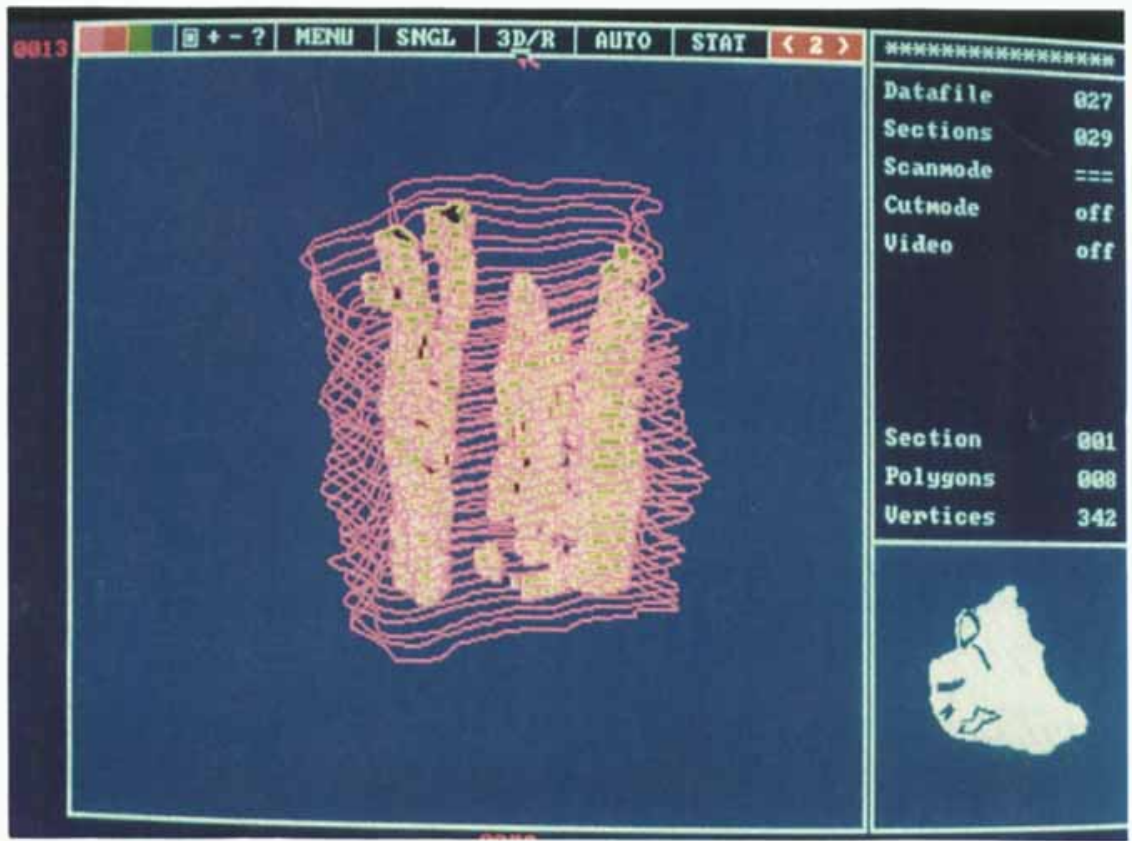

FIGURE 2. Glands with ramifications. Luminal structures are interconnected with one another (3-D).

Further studies are needed in order to evaluate if the two different types could be correlated either to the different degree of a "aggressiveness" or to the different appearances of peritoneal endometriosis.

From the present stereographic findings, one may consider that the apparently multifocal occurrence (in 2-D) of glandular epithelium in one lesion is not confirmed by the 3-D study. Indeed, in each peritoneal lesion, epithelial glands are interconnected by luminal structures. Probably, in each peritoneal lesion, epithelial structures occur in a single focus of the stroma and may then gradually develop, elongate and swell forming luminal structures, occasionally with endometrial debris inside. During the expansion, all glands are connected and no peripheral epithelial structures become independent by loss of interconnection.

Since each of the peritoneal lesions stereographically reconstructed in the

TABLE 3. Stereometry of Volumes of 3-D Structures: Percentage of the Lesion Attributed to the Epithelium, Stroma, or Lumen

\begin{tabular}{lcc}
\hline Ratio (\%) & Group I & Group II \\
$\mathrm{n}=26$ & $\mathrm{n}=16$ \\
\hline Epithelium/lesion & $19.9 \%$ & $14.9 \%$ \\
Stroma/lesion & $62.2 \%$ & $51.8 \%$ \\
Lumen/lesion & $13.2 \%$ & $25.2 \%$ \\
\hline
\end{tabular}


present study was only a part of the peritoneal endometriosis in one patient, it is far too soon to come to any conclusion, but in the future, the 3-D analysis of stereographically reconstructed lesions could contribute to the understanding of the in vivo development of endometriosis.

This stereometric study of volumes of 3-D structures revealed the volume distribution in peritoneal endometriosis. The ratios of epithelium, stroma, lumen/ lesion observed in group I and II indicate a stronger effect of the GnRH agonist therapy on the stroma than on the epithelium. This effect could be due to the reduction in stromal vascularization induced by $\mathrm{GnRH}$ agonist. The stromal capillary network can also be reconstructed in 3-D. In active lesions, the 3-D evaluation of the capillary network reveals the presence of a great number of larger vessels. After GnRH agonist therapy (as well as in white lesions), the network is composed of smaller vessels.

The present stereographic and stereometric study has shown some new characteristics of peritoneal endometriosis. Further studies will be required to investigate the variations in the 3-D architecture of peritoneal endometriotic lesions among the different appearances of endometriosis in individual patients, among patients of different ages, or among patients having different types of peritoneal endometriosis before and after hormonal therapy.

\section{REFERENCES}

Bergqvist, A., G. Rannevik \& J. Thorell. 1981. Estrogen and progesterone cytosol receptor concentration in endometriotic tissue and intrauterine endometrium. Acta Obstet. Gynecol. Scand. 101: 53-58.

BRAVERMAN, M. S. \& I. M. BRAVERMAN. 1986. Three-dimensional reconstructions of objects from serial sections using a microcomputer graphic system. J. Invest. Dermatol. 86: 290-294.

Brosens, I., G. Vasquez \& S. Gordts. 1984. Scanning electron microscopic study of the pelvic peritoneum in unexplained infertility and endometriosis. Fertil. Steril. 41: 215.

Chatman, D. L. 1981. Pelvic peritoneal defects and endometriosis; Allen-Masters syndrome revisited. Fertil. Steril. 36: 751.

Donnez, J., M. Nisolle \& F. Casanas-Roux. 1992. Three-dimensional architectures of peritoneal endometriosis. Fertil. Steril. 57: 980.

Ito, M., H. YokoYama, K. IKEDA \& Y. SAT. 1990. Stereographic analysis of syringomas. Arch. Dermatol. Res. 282: 17-21.

JANNE, O., A. KaUPPILA \& E. KoKKO. 1981. Estrogen and progestin receptors in endometriosis lesions: comparison with endometrial tissue. Am. J. Obstet. Gynecol. 141: 562-566.

JANSEN, R. P. S. \& P. RusSell. 1986. Nonpigmented endometriosis: clinical laparoscopic and pathologic definition. Am. J. Obstet. Gynecol. 155: 1154.

Lox, C. D., L. WORD \& M. W. HEINE. 1984. Ultrastructural evaluation of endometriosis. Fertil. Steril. 41: 755.

MarChevsky, A. M., J. Gil \& H. JeAnTY. 1987. Computerized interactive morphometry in pathology: current instrumentation and methods. Hum. Pathol. 18: 320-331.

Martin, D. C., G. D. Hubert, R. Vander Zwaag \& F. EI-Zfey. 1989. Laparoscopic appearances of peritoneal endometriosis. Fertil. Steril. 51: 63.

MatTa, W. H. M., I. Stabille, R. S. Shaw \& S. Camprell. 1988. Doppler assessment of uterine blood flow changes in patients with fibroids receiving the $\mathrm{GnRH}$ agonist Buserelin. Fertil. Steril. 49: 1083.

Murphy, A. A., W. R. Green, D. Bobbie, Z. C. de la Cruz \& J. A. Rock. 1986. Unsuspected endometriosis documented by scanning electron microscopy in visually normal peritoneum. Fertil. Steril. 46: 522 .

Nisolle, M., F. Casanas-Roux \& J. Donnez. 1988. Histologic study of ovarian endometriosis after hormonal therapy. Fertil. Steril. 49: 423. 
Nisolle, M., B. Paindaveine, A. Bourdon, M. Berlière, F. Casanas-Roux \& J. DonNez, 1990. Histologic study of peritoneal endometriosis in infertile women. Fertil. Steril. 53: 984-988.

Nisolle, M., F. Casanas-Roux, V. Anaf, J. M. Mine \& J. Donnez. 1993. Morphometric study of the stromal vascularization in peritoneal endometriosis. Fertil. Steril. 59: 681.

REDWINE, D. B. 1987. The distribution of endometriosis in the pelvis by age groups and fertility. Fertil. Steril. 47: 173-175.

RODDICK, J. W., G. CONKEY \& E. J. JACOBS. 1960. The hormonal response of endometriotic implants and its relationship to symptomatology. Am. J. Obstet. Gynecol. 79: 1173-1177.

Stripling, M. C., D. C. Martin, D. L. Chatman, R. Vander Zwaag \& W. M. Poston. 1988. Subtle appearances of pelvic endometriosis. Fertil. Steril. 49: 427.

Tamaya, T., T. Motoyaha \& Y. OHONo. 1979. Steroid receptor levels and histology of endometriosis and adenomyosis. Fertil. Steril. 31: 394-400. 